\section{Are you sitting comfortably?}

Most of us, without even realising it, are damaging our spines by the way we sit at work. For dental professionals, this is a serious issue, since musculoskeletal disorders are the main reasons for early retirement in the profession.

In other words, simply sitting down could be affecting the quality of your work and cutting years off your healthy career.

A-dec has a solution. Its range of ergonomic stools, including the premier A-dec 500 range, have been specially designed to provide dental professionals with an effective and comfortable seating option. A dynamic system, the A-dec 500 stool has three layers of flexible material designed to conform to your every shift and movement, providing you with unprecedented support and comfort.

What's more, the stool features for points of adjustability, enabling you to find the correct posture - and precise increments of movement can be made easily throughout the day to ensure you are sitting comfortably no matter what.

The A-dec 500 stool also features a variety of innovative optional features that increase its ergonomic efficacy. From additional foot pedestals to encourage the healthier 'athletic' stance to swing-out armrests for optimum support, the stool has everything you need to practise dentistry safely and comfortably.

Combining A-dec's iconic durability with effortless stylishness and functionality, the A-dec 500 stool is the perfect addition to any modern dental surgery. Contact the expert team today to try it for yourself. Visit www.a-dec.co.uk or call 0800233285.
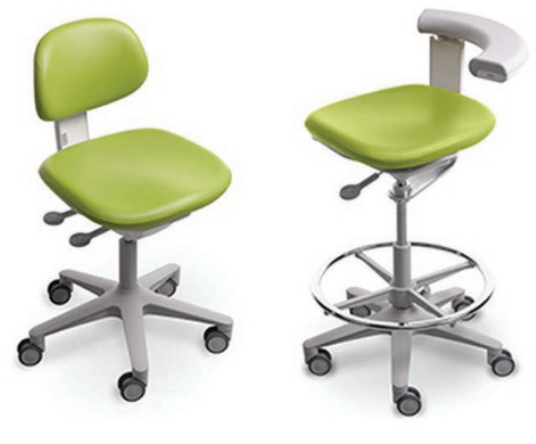

\title{
An effective alternative to wire interdental brushes
}

Do some of your patients struggle with wire interdental brushes? Are you looking for an effective alternative you can trust to recommend to them?

Then look no further than Wisdom Clean Between Rubber Interdental Brushes. The highly popular products feature a flexible, tapered design with unique and super soft micro-fine filaments that glide easily between the teeth, around orthodontic appliances or around crowns or bridges. Removing food debris and plaque as they go, the brushes are clinically proven to reduce gingival disease, ensuring a thorough yet gentle clean while also massaging the gingiva.

What's more, they are completely wire-free and provide an ideal solution for patients who find wire interdental brushes uncomfortable or challenging to use correctly. Available in three sizes, the brushes are ideal for use in interdental spaces of various dimensions.

The whole range of Wisdom products includes the Wisdom Super Slim Interspace Brush, Wisdom Clean Between Easy Slide Y Shaped Floss Harps with PTFE tape and Wisdom Easy Flosser also with PTFE tape.

To find out more, visit www.wisdomtoothbrushes.com or call 01440714800 .

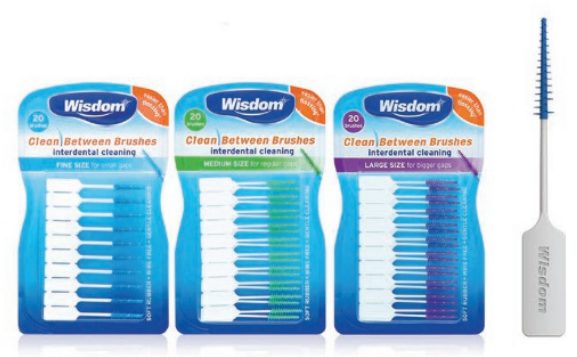

\section{Ethical anterior alignment training and appliances}

Dedicated to offering ethical anterior alignment training and appliances, the IAS Academy is pleased to announce the launch of the ClearSmile Aligner 2.0.

Building upon the existing excellence of the original ClearSmile Aligner appliance, the

2.0 offers a greater level of predictability and accuracy of movement for even better results.

There have been a number of changes made from the original design, all of which the

Academy expects will facilitate a higher standard of anterior alignment training with quality outcomes that both patient and practitioner can be proud of.

The key benefits of the ClearSmile Aligner 2.0 include:

- Virtual digital planning for predictable outcomes

- Precise 3D manufacturing

- Exclusive stretch-resistant intelligent material

- Biomechanical indices for ideal
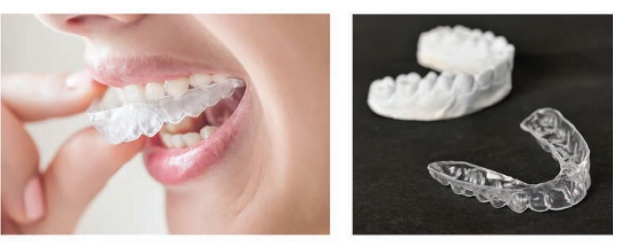
staging

- Custom Force Drivers for individual tooth control.

Visit www.iasortho.com or call 02089162024.

\section{Accurate. Mobile. Simple!}

The Mobile True Definition Scanner from $3 \mathrm{M}$ Oral Care is truly one of a kind. As the world's very first mobile intraoral scanner that operates solely on a tablet, the Mobile True Definition affords practitioners with a flexible diagnostic tool.

Because it is easy to move and fits perfectly in yours or your patients' hands, the Mobile True Definition system can be used as a powerful educational tool. Patients can be shown a clear and easy-to-understand representation of their oral anatomy, leading to increased patient acceptance.

What's more, the Mobile True Definition is more accurate than other leading scanners, and is appropriate for a wide range of indications, including crowns and bridges, inlays and onlays, veneers, implants, partials and even orthodontic appliances.

To find out more, contact the expert team at 3M Oral Care today on 08456025094 or visit www.3Mespe.co.uk. 